\title{
Soft X-Ray Imaging
}

\author{
John Seely, Charles Brown, and Glenn Holland \\ Space Science Division, Nayal Research Laboratory \\ Code 7674, Washington DC 20375-5352 \\ Tel. 202-767-3529, john.seely@nrl.navy.mil
}

\section{Contents:}

1. Design of the Soft X-Ray Telescope

2. Fabrication and Characterization of the Soft X-Ray Telescope

3. Experimental Implementation at the OMEGA Laser Facility

\section{Design of the Soft X-Ray Telescope}

The telescope is a Cassegrain design with a concave primary mirtor and a convex secondary mirror. The mirrors have multilayers coutings that reflect $48 \AA(260 \mathrm{eV})$ radiation from the laser-produced plasma. The secondary mirtor is divided into four individually adjustable sectors which project four images to the framing camera. The magnification factor is X12. Sketches of the telescope and the secondary mitor mount are shown in Fig. 1.
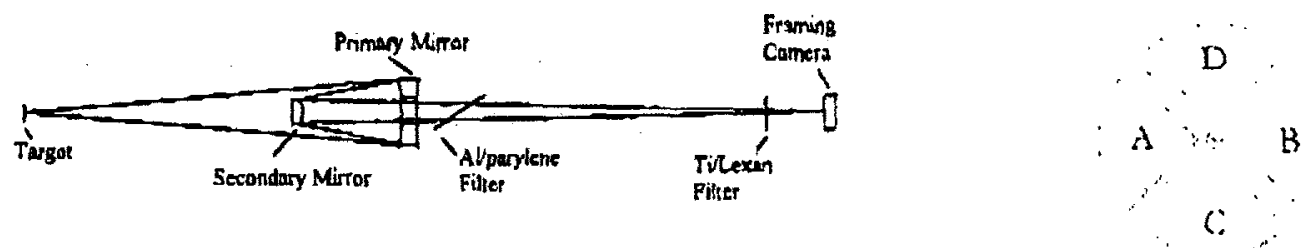

Pig. 1. Skctch of the telescope (left, not to scale) and the sccondary mirror mount (right) showing the orientation of the four sectors of the secondary mirror and the arrangement of the $3 \mathrm{~mm}$ gaps between the sectors. Also indicated is an axjal alignment hole.

The secondary mirror mount is attached to a spider support and that has a tilutip fine adjustment. A ball bearing centered in two conical holes provides a fixed pivot for this tilt/tip mechanism. It is remotely adjustable by two MotorMike drives.

Small diameter centering holes are available in each optical clement/support to allow a laser beam to propagate through the instriment on its axis. These holes are threaded to allow closure with a screw. A closely fitted plug with a centering hole is provided for the center of the primary. A diode laser was then aligned to the instrument axis. The tilt and tip of each optical mount was approximately adjusted beforc mounting the optics by retroreflecring the axial laser beam on itself. 


\section{DISCLAIMER}

This report was prepared as an account of work sponsored by an agency of the United States Government. Neither the United States Government nor any agency thereof, nor any of their employees, make any warranty, express or implied, or assumes any legal liability or responsibility for the accuracy, completeness, or usefulness of any information, apparatus, product, or process disclosed, or represents that its use would not infringe privately owned rights. Reference herein to any specific commercial product, process, or service by trade name, trademark, manufacturer, or otherwise does not necessarily constitute or imply its endorsement, recommendation, or favoring by the United States Government or any agency thereof. The views and opinions of authors expressed herein do not necessarily state or reflect those of the United States Government or any agency thereof. 


\section{DISCLAIMER}

Portions of this document may be illegible in electronic image products. Images are produced from the best available original document. 
The Beam-4 optical ray tracing computer code was used to optimize the optical system. The following Table in Beam-4 format gives the essential optical parameters of the instniment. Only one quadrant is represented, but the other three are identical by symmetry.

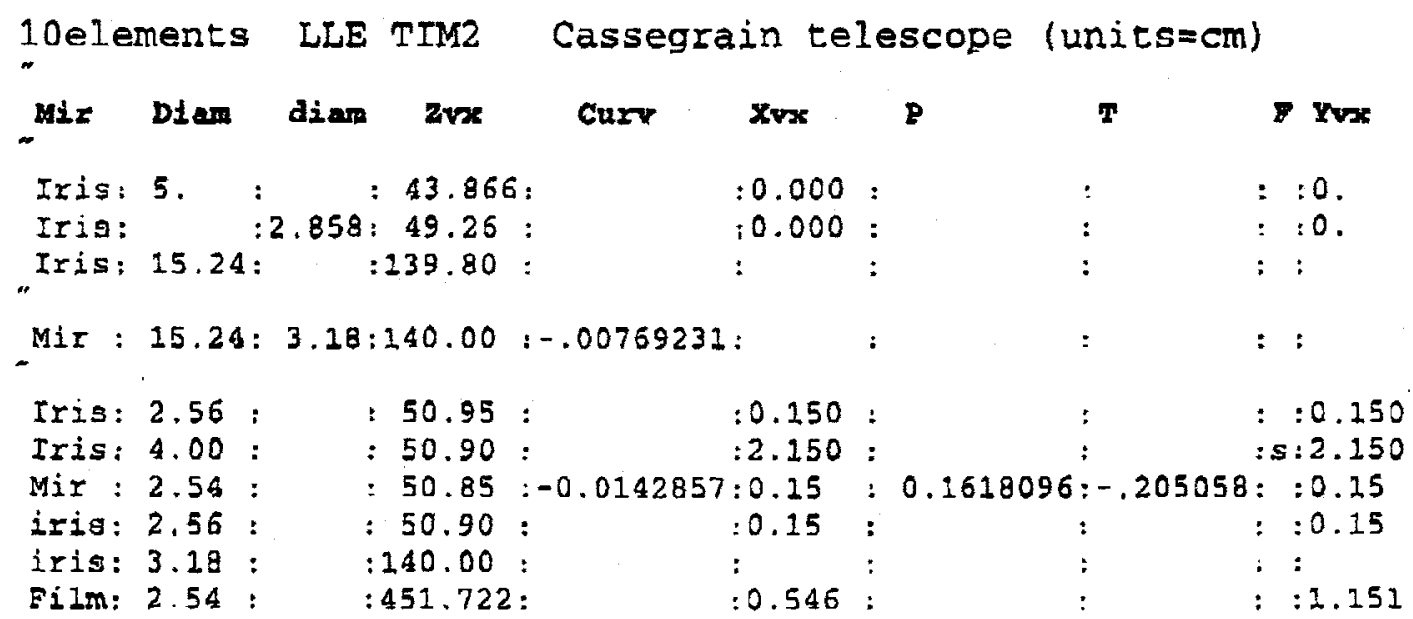

In this table, Diam is the outer diameter of an optic, diam is the diameter of a central obstruction, $\mathbf{X v x}, \mathbf{Y v x}$, and $\mathbf{Z v x}$ are the location of the center of the optic in $\mathrm{cm}$, Curv is the reciprocal of the radius of curvature, and $\mathbf{P}$ and $\mathbf{T}$ are the pitch and tilt in degrees, respectively. $\mathbf{F}$ is a shape parameter, $s$ denoting a square or rectangular element. The source point is at $X=Y=Z=0$. The "film" is given $X$ and $Y$ coordinates corresponding to a selected strip on the framing camera.

Fig. 2. The spor diagram enlculared by the Betrm-4 rntical ray tracing code. This is the spot diagram of one quadrant of the optical system. The other three sectors yield similar dingrams, rotated by 90 degrees, 180 degrees, and 270 degrees.

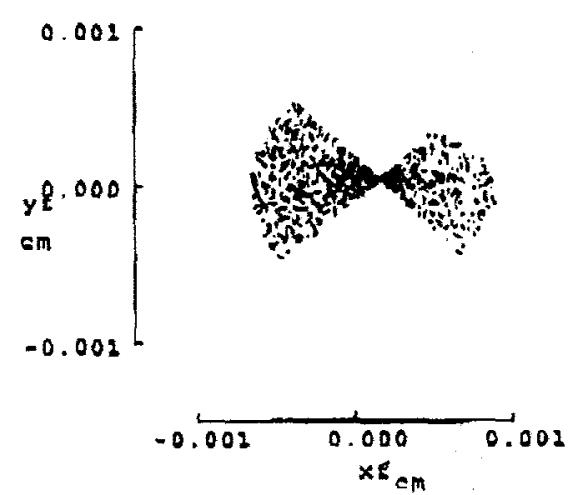

The spor diagram is shown in Fig. 2. The spor size is upproximately $5 \mu \mathrm{m}$ in the vertical direction and $10 \mu \mathrm{m}$ in the horizontal direction. This corresponds to a spatial resolution $<1 \mu \mathrm{m}$ in the object plane. During the alignment and focusing of the telescope using a mesh object and visible light, it was noticed that sectors $A$ and $B$ gave better focus for horizontal wires while $C$ and $D$ were betrer for vertical wires. This was consistent with the ray trace study, where the comatic image of a point was elongated horizontally for $A$ and $B$ and vertically for $C$ and $D$. 
Fig. 3. The positions of the four images on the framing camera photocathode.

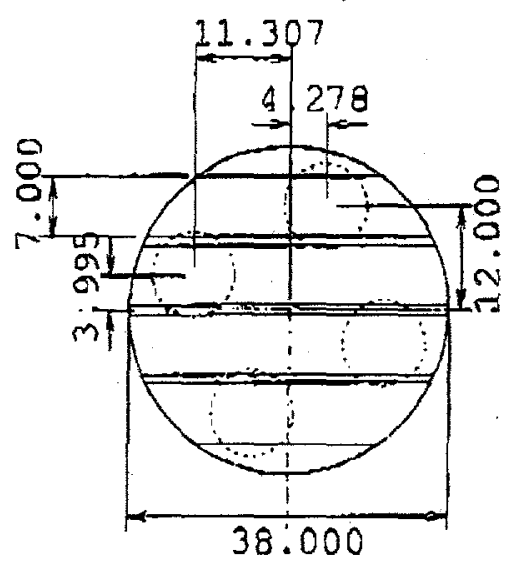

Figure 3 is a diagram of the four focal spots on the four-strip framing camera photocathode. The spots are arranged so that one spot falls on each of the four strips. Images are recorded at four different times by gating the strips on the MCP.

Three primary mirrors and three cut secondary mirror sets were procured from General Optics Inc. The mirrors were superpolished to a microroughness of 1 . One primary mirror and one secondary mirror set were sent to OSMIC Inc for deposition of the multilayer coatings. Another secondary mirror set was coated with aluminum and used along with an uncoated primary for optical alignment. The third secondary mirror sel and the third primary mirror were held as backups. The backup mirrors were eventually multilayer coated following the Rochester experiments.

The multilayer coating was specified to have peak reflectance at a wavelength of $48.3 \AA$. This wavelength was chosen because of the excellent results obtained from previous experiments at LLE using a multilayer-coated telescope producing one $\mathrm{X} 6$ magnified image at a wavelength of $48.3 \mathrm{~A}$.' The coating consisted of altemating layers of chromium and carbon. Based on the previous results, a peak reflectance of at least $6 \%$ was expected.

Owing to unexpected delays in the delivery of the multilayer-coated mirrors, there was not time before the experiment to measure the reflectance of the coated mirrors. After the experiment, the backup mirrors were couted, and the reflectance of one of the secondary mirrors was measured using the NRL beamline at the Brookhaven synchrotron. For an angle of incidence of $10^{\circ}$, the measured peak reflectance was $12 \%$ at a wavelength of $47.1 \AA$. This corresponds 10 a normal-incidence peak wavelength of 47.9 A which is suitably close to the desired wavelength of $48.3 \mathrm{~A}$.

\section{Fabrication and Characterization of the Soft X-Ray Telescope}

The primary mirror is made of superpolished zerodur on a 6 " OD blank and has a $1.25^{\prime \prime}$ central opening. The radius of curvature is $130 \mathrm{~cm}$. It is mounted in an aluminum cup with three manual tip/tilt adjuster screws and a MotorMike encoded micrometer driving a linear focus adjusiment. The linear movement is controlled by three ball bushings riding on two precision shafrs. 
The zerodor secondary mirtor was sawed into four parts, rejoined and superpolished as a single unit to the same radius $(70 \mathrm{~cm})$. These sectors were glued with EpoTek 301 epoxy to a four-segment flexure mechanism with three glue pads for each mirror sector. The four sectors are individually adjusted with four $\$ 0-80$ screws paired in a push-pull arrangement. While the original secondary was l" in diameter, the four sectors were mounted with a $0.125^{\prime \prime}$ gap between the individual sectors. This gap was determined by ray tracing to optimize the secondary's acceptance angle, while negligibly degrading the optical performance with expected detectors.

Two 0.125 " rods were notched in the center and joined in a cross to form a centering jig for gluing the mirror sectors, EpoTek 301 was weighed out (4 gm resin \& 1 gm hardener) and stirred for $15 \mathrm{~min}$. A small drop of glue was transferred to each glue pad, and the mirror sector carefully lowered into place and pushed against the centering cross. The glue was of low viscosity, and gravity served to hold the sectors in place during curing of the epoxy. The glued secondary was allowed to sit undisturbed for $24 \mathrm{hr}$.

The setup optics were installed in the instrument at the optimal locations as indicated by the ray tracing, and a target mesh located at the object position. It was back illuminated by the image of a fiber optic illuminator formed by two $19 \mathrm{~cm}$ focal length lenses. The fiber optic was placed at the focus of one lens, and the target mesh at the focus of the other. Approximately parallel light passed from one lens to the other, so the distance between them could be easily varied. This same arrangement was eventually set up in TMM5 opposite TIM2 at LLE. The lenses were $-50 \mathrm{~mm}$ in diameter, so their $\mathrm{f}$ number $(-5)$ easily overfilled the primary mirror.

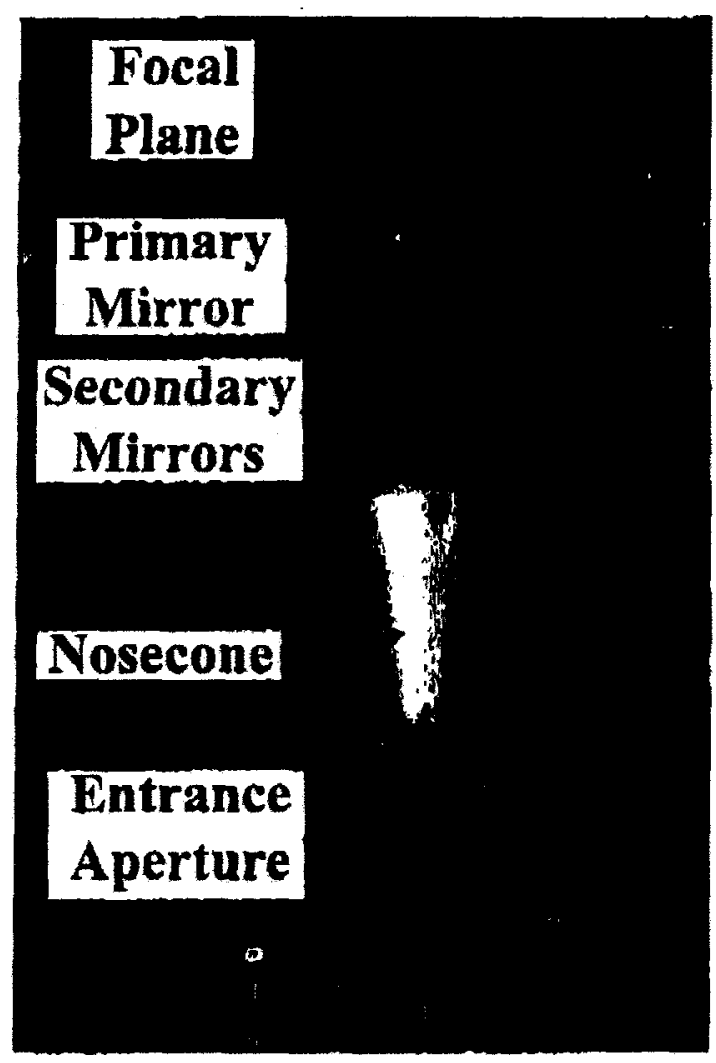

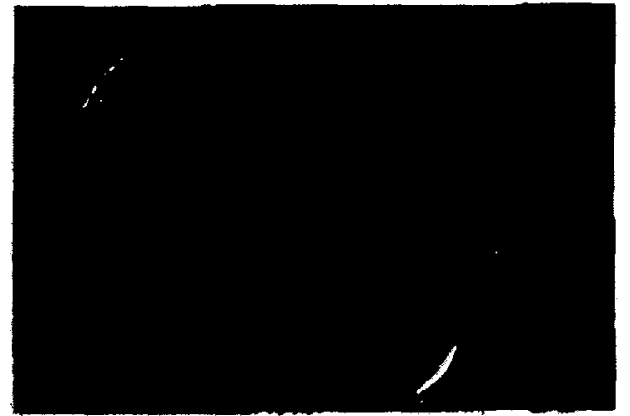

Pig. 4. The assembled telescope (leit) and the four images projected onto the pocal-plane screen (above). The lines on the screen indicate the four strips of the framing camera (below).

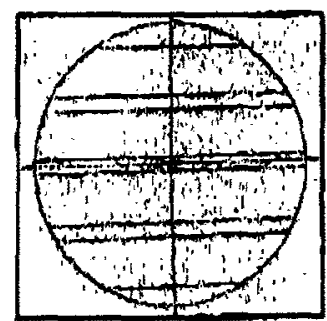


A focusing screen was placed at the detector position, and a transparency showing the framing camera strips and desired image positions was taped to the screen. The flexure was adjusted to guide beams from each of the four secondaries to the desired spots on the screen. The primary minor was adjusted to center the illumination on the secondaries equally, and to give the best average focus. Photographs of the assembled telescope, the screen, and the four images on the screen are shown Fig. 4.

Shown in Fig. 5 is an enlargement of the images recorded with the room darkened. The object is an SEM specimen finder grid. A fiducial is located at the center of the grid, and this fiducial is clearly visible at the centers of the four images. The fiducial is supported on a mesh of $25 \mu \mathrm{m}$ wires which are also visible in the images. This mesh supported a finer $400 /$ inch mesh of $15 \mu \mathrm{m}$ wires. These $15 \mu \mathrm{m}$ wires were clearly visible when viewing the screen with the eye but are just barely visible in Fig. 5. While diffraction adversely affected the visible light images, diffraction is negligible for $48 \AA$ radiation imaged during the laser experiments at OMEGA.

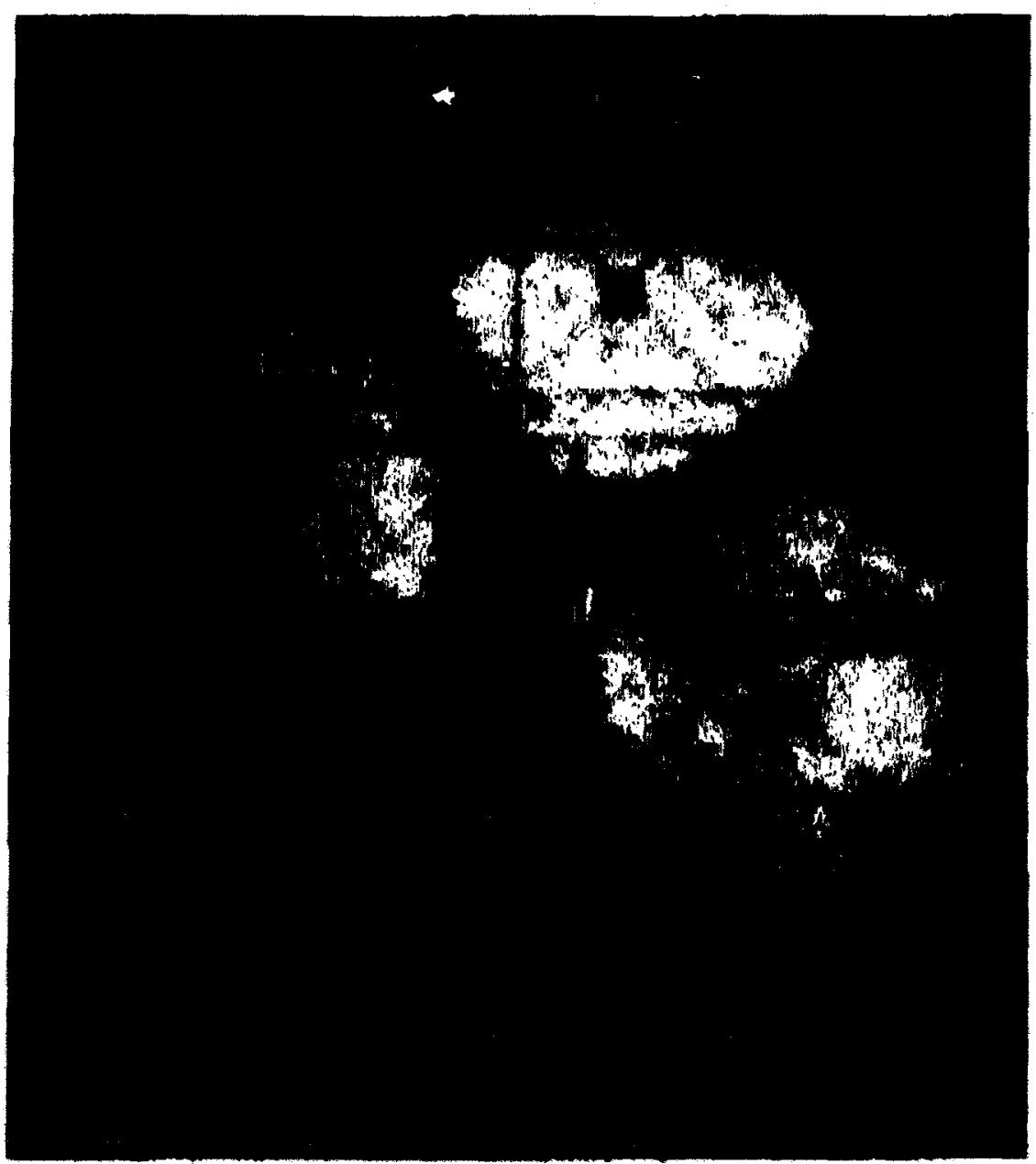

Fig. 5. Tho four images of an SEM specimen finder grid. Visible in cach image are the central fiducial and the coarse $(25 \mu \mathrm{m})$ and the fine $(15 \mu \mathrm{m})$ wire patterns. 


\section{Experimental Implementation at the OMEGA Laser Facility}

Using an aluminum box that simulated a TMM boat, the telescope was assembled and aligned at NRL in the two weeks prior to the trip to Rochester. The aluminum box also served as a support for the telescope in the shipping case.

The coated mirrors were installed in the telescope the weekend before the experiments (the set-ups were done with an uncoated primary and an aluminum coated secondary mirror assembly). Target chamber alignment techniques were developed.

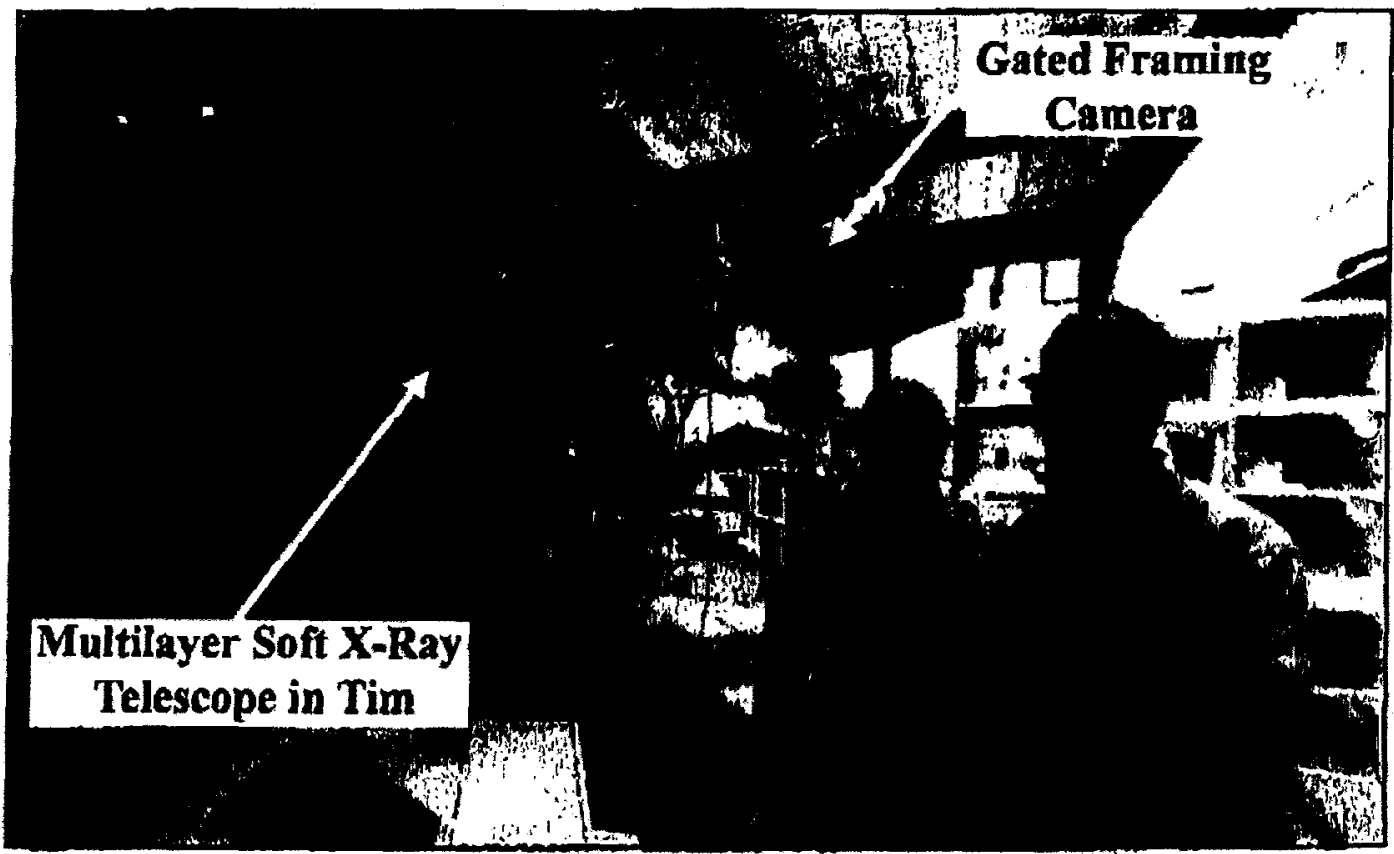

Fig. 6. The multilayer telescope was mounted in TIM2 on the OMEGA target chamber, and the gated framing camera was mounted to a small vact um chamber attached to the end of TIM2.

As shown in Fig. 6, the multilayer telescope was fielded in TIM2, and the framing camera was attached to the end of TLM2. Data were recorded on five shots on May 27, 1998. On the first shot, the framing camera was set up to activate the four strips at intervals of $0.4 \mathrm{nsec}$ during the laser shot. The target consisted of an SEM grid illuminated by a gold backlighter $9 \mathrm{~mm}$ behind the grid. A 500 \& thick Formvar screen was positioned midway between the backlighter and the grid. The backlighter was illuminated by 11 beams and $2 \times 10^{14} \mathrm{~W} / \mathrm{cm}^{2}$. Upon inspection of the framing camera film after that shot, the shadow of a retaining clip was identified on the film. No grid pattern was seen on the film. Apparently, the clip was left in the camera head during setup by LLE staff, and it is likely that this clip caused the camera to malfunctioned on the first laser shot. The clip was removed from the camera.

Another laser shot was taken with similar experimental conditions, and no grid pattern was seen on the film. After this shot, the alignment of the telescope was checked by mounting the visible light fixture in TIM5 opposite the telescope's TIM2. The four images fell in the proper positions for recording by the framing camera. 
On the next shot, the Formvar screen was removed, and on the following shot the framing camera was operated in time-integrating mode. No grid pattem was observed on either shot. On the final shot, the framing camera was replaced by Kodak $101 \mathrm{film}$, and no grid pattern was observed.

Following the experiments, the alignment was again checked using visible light, and the images were properly positioned in the focal plane. However, inspection of the primary minor revealed that the coating was severely ablated in the regions that were directly exposed to the target plasma.

The causes of the negative experimental results were inferred to be the following: The clip that was inadventently left in the framing camera caused the camera to malfunction beginning with the first shot. Also beginning on the first shot, the multilayer coating was severely ab!ated by the radiation and debris fiux from the target. The reflectance of the multilayer coating was likely completely destroyed in the damaged regions. The low reflectance of the multilayer coatings, damaged on the first laser shot, reduced the exposure on the framing camera below the detection threshold.

'J. Seely, G. Holland, T. Boehly, G. Pien, and D. Bradley, Applied Optics 37, 1140 (1998). 